\title{
Product Intuitionistic Fuzzy Graph
}

\author{
N. Vinoth Kumar \\ Assistant Professor \\ M.A.M. School of Engineering, \\ Trichy, Tamilnadu \\ India- 621105
}

\author{
G. Geetha Ramani \\ Assistant professor, Department of Mathematics \\ Anna University of Technology \\ Trichy, Tamilnadu \\ India- 620024
}

\begin{abstract}
In this paper, we introduce product intuitionistic fuzzy graphs and prove several results which are analogous to intuitionistic fuzzy graphs. We conclude by giving properties for a product partial intuitionistic fuzzy sub graph.
\end{abstract}

Key words: Intuitionistic Fuzzy Graphs, Product Intuitionistic fuzzy graphs.

\section{INTRODUCTION}

The first definition of fuzzy graphs was proposed by Kafmann, from the fuzzy relations introduced by Zadeh. Although Rosenfeld [1] introduced another elaborated definition, including fuzzy vertex and fuzzy edges, and several fuzzy analogs of graph theoretic concepts such as paths, cycles, connectedness and etc. The first definition of intuitionistic fuzzy graphs was proposed by Atanassov[2]. Dr. V. Ramaswamy and Poornima .B introduce the concept of product fuzzy graph. In this paper we develop the concept of Product Intuitionistic fuzzy graphs of intuitionistic fuzzy graphs. Further investigate properties Product Intuitionistic fuzzy graphs.

\section{DEFINITION AND MAIN RESULTS}

Definition 2.1 An intuitionistic fuzzy graph (IFG) is of the form $\mathrm{G}=(\mathrm{V}, \mathrm{E})$, where $\mathrm{V}=\left\{\mathrm{v}_{1}, \mathrm{v}_{2}, \ldots, \mathrm{v}_{\mathrm{n}}\right\}$ such that $\mu_{1}: \mathrm{V} \rightarrow[0,1]$, $\gamma_{1}: \mathrm{V} \rightarrow[0,1]$ denote the degree of membership and nonmember ship of the element $v i \in V$ respectively and $0 \leq \mu_{1}\left(v_{i}\right)+\gamma_{1}\left(v_{i}\right) \leq 1$ for every $\mathrm{v}_{\mathrm{i}} \in \mathrm{V}$,(i $\left.=1,2, \ldots \mathrm{n}\right)$ (ii) $\mathrm{E} \subseteq \mathrm{V} \times \mathrm{V}$ where $\mu_{2}: \mathrm{V} \times \mathrm{V} \rightarrow[0,1]$ and $\gamma_{2}: \mathrm{V} \times \mathrm{V} \rightarrow[0,1]$ are such that $\mu_{2}\left(\mathrm{v}_{\mathrm{i}}, \mathrm{v}_{\mathrm{j}}\right) \leq \mu_{1}\left(\mathrm{v}_{\mathrm{i}}\right) \wedge \mu_{1}\left(\mathrm{v}_{\mathrm{j}}\right), \gamma_{2}\left(\mathrm{v}_{\mathrm{i}}, \mathrm{v}_{\mathrm{j}}\right) \leq \gamma_{1}\left(\mathrm{v}_{\mathrm{i}}\right) \wedge \gamma_{1}\left(\mathrm{v}_{\mathrm{j}}\right)$ and $0 \leq \mu_{2}\left(\mathrm{v}_{\mathrm{i}}, \mathrm{v}_{\mathrm{j}}\right)+\gamma_{2}\left(\mathrm{v}_{\mathrm{i}}, \mathrm{v}_{\mathrm{j}}\right) \leq 1$.

Definition 2.2 Let $\mathrm{G}=(\mathrm{V}, \mathrm{E})$ be an intuitionistic fuzzy graph. If $\mu_{2}(\mathrm{x}, \mathrm{y}) \leq \mu_{1}(\mathrm{x}) \times \mu_{1}(\mathrm{y})$ and $\gamma_{2}(\mathrm{x}, \mathrm{y}) \leq \gamma_{1}(\mathrm{x}) \times \gamma_{1}(\mathrm{y})$ the intuition fuzzy graph is called product partial intuitionistic fuzzy sub graph of G.

Remark: If $\mathrm{G}=(\mathrm{V}, \mathrm{E})$ is a product intuitionistic fuzzy graph then since $\mu_{1}(\mathrm{x})$ and $\mu_{1}(\mathrm{y})$, are less than or equal to 1 , it follows that $\mu_{2}\left(\mathrm{v}_{\mathrm{i}}, \mathrm{v}_{\mathrm{j}}\right) \leq \mu_{1}\left(\mathrm{v}_{\mathrm{i}}\right) \times \mu_{1}(\mathrm{vj}) \leq \mu_{1}\left(\mathrm{v}_{\mathrm{i}}\right) \wedge \mu_{1}\left(\mathrm{v}_{\mathrm{j}}\right)$ and $\gamma_{2}\left(\mathrm{v}_{\mathrm{i}}, \mathrm{v}_{\mathrm{j}}\right) \leq$ $\gamma_{1}\left(v_{i}\right) \times \gamma_{1}\left(v_{j}\right) \leq \gamma_{1}\left(v_{i}\right) \wedge \gamma_{1}\left(v_{j}\right)$ for all $x, y \in V$. Thus every product intuitionistic fuzzy graph is an intuitionistic fuzzy graph.

Definition 2.3 A product Intuitionistic fuzzy graph $\mathrm{G}=(\mathrm{V}, \mathrm{E})$ is said to be complete if $\mu_{2}(\mathrm{x}, \mathrm{y})=\mu_{1}(\mathrm{x}) \times \mu_{1}(\mathrm{y})$ and $\gamma_{2}(x, y)=\gamma_{1}(x) \times \gamma_{1}(y)$ for all $x, y \in V$.
Proposition 2.1 Let $\mathrm{G}=(\mathrm{V}, \mathrm{E})$ be a complete product intuitionistic fuzzy graph where $\mu_{1}$ and $\gamma_{1}$ are normal. Then $\mu_{2}{ }^{n}(x, y)=\mu_{2}(x, y)$ and $\gamma_{2}{ }^{n}(x, y)=\gamma_{2}(x, y)$ for all $x, y \in V$ and for all positive integer $\mathrm{n}$ for $\mathrm{n} \geq 2$

$$
\begin{aligned}
& \mu_{2}{ }^{n}(x, y)=\vee_{z \in V}\left\{\mu_{2}{ }^{n-1}(x, y) \times \mu_{2}(x, y)\right\} \\
& \gamma_{2}{ }^{n}(x, y)=v_{z \in V}\left\{\gamma_{2}{ }^{n-1}(x, y) \times \gamma_{2}(x, y)\right\}
\end{aligned}
$$

Proof: We prove by method of induction .Let $\mathrm{n}=2$ then for all $\mathrm{x}, \mathrm{y} \in \mathrm{V}$, we have

$$
\begin{aligned}
& \mu_{2}^{2}(\mathrm{x}, \mathrm{y})=\vee_{\mathrm{z} \in \mathrm{V}}\left\{\mu_{2}(\mathrm{x}, \mathrm{z}) \times \mu_{2}(\mathrm{z}, \mathrm{y})\right\} \\
& \mu_{2}^{2}(\mathrm{x}, \mathrm{y})=\vee_{\mathrm{z} \in \mathrm{V}}\left\{\left[\mu_{1}(\mathrm{x}) \times \mu_{1}(\mathrm{z})\right] \times\left[\mu_{1}(\mathrm{z}) \times \mu_{1}(\mathrm{y})\right]\right\} \\
& \mu_{2}^{2}(\mathrm{x}, \mathrm{y})=\vee_{\mathrm{z} \in \mathrm{V}}\left\{\mu_{1}(\mathrm{x}) \times \mu_{1}(\mathrm{y}) \times \mu_{1}(\mathrm{z})^{2}\right\} \\
& \text { Since } \mu_{1}(\mathrm{z})^{2} \leq 1 \text { for all } \mathrm{z}\left[\therefore \mu_{1}(\mathrm{z}) \leq 1\right] \\
& \qquad \mu_{2}^{2}(\mathrm{x}, \mathrm{y})=\vee_{\mathrm{z} \in \mathrm{V}}\left\{\mu_{1}(\mathrm{x}) \times \mu_{1}(\mathrm{y})\right\} \\
& \mu_{2}^{2}(\mathrm{x}, \mathrm{y})=\mu_{2}(\mathrm{x}, \mathrm{y}) \rightarrow(1) \\
& \text { and } \quad \gamma_{2}^{2}(\mathrm{x}, \mathrm{y})=\mathrm{v}_{\mathrm{z} \in \mathrm{V}}\left\{\gamma_{2}(\mathrm{x}, \mathrm{z}) \times \gamma_{2}(\mathrm{z}, \mathrm{y})\right\} \\
& \gamma_{2}^{2}(\mathrm{x}, \mathrm{y})=\vee_{\mathrm{z} \in \mathrm{V}}\left\{\left[\gamma_{1}(\mathrm{x}) \times \gamma_{1}(\mathrm{z})\right] \times\left[\gamma_{1}(\mathrm{z}) \times \gamma_{1}(\mathrm{y})\right]\right\} \\
& \gamma_{2}^{2}(\mathrm{x}, \mathrm{y})=\vee_{\mathrm{z} \in \mathrm{V}}\left\{\gamma_{1}(\mathrm{x}) \times \gamma_{1}(\mathrm{y}) \times \gamma_{1}(\mathrm{z})^{2}\right\} \\
& \text { since } \gamma_{1}(\mathrm{z})^{2} \leq 1 \text { for all } \mathrm{z}\left[\therefore \gamma_{1}(\mathrm{z}) \leq 1\right] \\
& \qquad \gamma_{2}^{2}(\mathrm{x}, \mathrm{y})=\vee_{\mathrm{z} \in \mathrm{V}}\left\{\gamma_{1}(\mathrm{x}) \times \gamma_{1}(\mathrm{y})\right\} \\
& \gamma_{2}^{2}(\mathrm{x}, \mathrm{y})=\gamma_{2}(\mathrm{x}, \mathrm{y}) \rightarrow(2)
\end{aligned}
$$

If $\mu_{1}$ and $\gamma_{1}$ normal, then $\mu_{1}(t)=1$ and $\gamma_{1}(t)=1$ for some $t$. Then

$$
\begin{aligned}
\mu_{2}^{2}(\mathrm{x}, \mathrm{y}) & =\vee_{\mathrm{z} \in \mathrm{V}}\left\{\mu_{1}(\mathrm{x}) \times \mu_{1}(\mathrm{y}) \times \mu_{1}(\mathrm{z})^{2}\right\} \\
& \geq \mu_{1}(\mathrm{x}) \times \mu_{1}(\mathrm{y}) \times \mu_{1}(\mathrm{t})^{2} \\
& =\mu_{1}(\mathrm{x}) \times \mu_{1}(\mathrm{y}) \quad\left[\therefore \mu_{1}(\mathrm{t})^{2}=1\right] \\
\mu_{2}^{2}(\mathrm{x}, \mathrm{y}) & \geq \mu_{1}(\mathrm{x}) \times \mu_{1}(\mathrm{y})
\end{aligned}
$$

$\mu_{2}(\mathrm{x}, \mathrm{y}) \geq \mu_{2}(\mathrm{x}, \mathrm{y}) \rightarrow(3)$ [since $\mu_{2}^{2}(\mathrm{x}, \mathrm{y})=\mu_{1}(\mathrm{x}) \times \mu_{1}(\mathrm{y}) \quad \mathrm{G}$ is complete]

from (1) and (3) we get $\mu_{2}(\mathrm{x}, \mathrm{y})=\mu_{2}(\mathrm{x}, \mathrm{y}) \rightarrow$ (4)

If $\gamma_{1}$ normal, $\gamma_{1}(\mathrm{t})=1$ for some $\mathrm{t}$. Then

$$
\begin{aligned}
\gamma_{2}^{2}(\mathrm{x}, \mathrm{y}) & =\vee_{\mathrm{z} \in \mathrm{V}}\left\{\gamma_{1}(\mathrm{x}) \times \gamma_{1}(\mathrm{y}) \times \gamma_{1}(\mathrm{z})^{2}\right\} \\
& \geq \gamma_{1}(\mathrm{x}) \times \gamma_{1}(\mathrm{y}) \times \gamma_{1}(\mathrm{t})^{2} \\
& =\gamma_{1}(\mathrm{x}) \times \gamma_{1}(\mathrm{y}) \quad\left[\therefore \mu_{1}(\mathrm{t})^{2}=1\right] \\
\gamma_{2}^{2}(\mathrm{x}, \mathrm{y}) & \geq \gamma_{1}(\mathrm{x}) \times \gamma_{1}(\mathrm{y})
\end{aligned}
$$

$\gamma_{2}(\mathrm{x}, \mathrm{y}) \geq \gamma_{2}(\mathrm{x}, \mathrm{y}) \rightarrow(5) \quad\left[\right.$ since $\gamma_{2}^{2}(\mathrm{x}, \mathrm{y})=\gamma_{1}(\mathrm{x}) \times \gamma_{1}(\mathrm{y}), \mathrm{G}$ is complete]

from (2) and (5) we get $\gamma_{2}(x, y)=\gamma_{2}(x, y) \rightarrow(6)$

Now assuming that $\mu_{2}{ }^{\mathrm{k}}(\mathrm{x}, \mathrm{y})=\mu_{2}(\mathrm{x}, \mathrm{y})$ and $\gamma_{2}{ }^{\mathrm{k}}(\mathrm{x}, \mathrm{y})=\gamma_{2}(\mathrm{x}, \mathrm{y})$ we will prove $\mu_{2}{ }^{\mathrm{k}+1}(\mathrm{x}, \mathrm{y})=\mu_{2}(\mathrm{x}, \mathrm{y})$ and $\gamma_{2}{ }^{\mathrm{k}+1}(\mathrm{x}, \mathrm{y})=\gamma_{2}(\mathrm{x}, \mathrm{y})$ we have

$\mu_{2}{ }^{\mathrm{k}+1}(\mathrm{x}, \mathrm{y})=\mathrm{v}_{\mathrm{z} \in \mathrm{V}}\left\{\mu_{2}{ }^{\mathrm{k}}(\mathrm{x}, \mathrm{z}) \times \mu_{2}(\mathrm{z}, \mathrm{y})\right\}$

$\mu_{2}^{2}(\mathrm{x}, \mathrm{y})=\vee_{\mathrm{z} \in \mathrm{V}}\left\{\left[\mu_{2}(\mathrm{x}, \mathrm{z}) \times \mu_{2}(\mathrm{z}, \mathrm{y})\right]\right\}=\mu_{2}^{2}(\mathrm{x}, \mathrm{y})$

$\mu_{2}^{\mathrm{k}+1}(\mathrm{x}, \mathrm{y})=\mu_{2}(\mathrm{x}, \mathrm{y}) \mathrm{using}(5)$

Similarly we get $\gamma_{2}{ }^{\mathrm{k}+1}(\mathrm{x}, \mathrm{y})=\gamma_{2}(\mathrm{x}, \mathrm{y})$

Hence proved 
Definition 2.4 The complement of product intuitionistic fuzzy graph $\mathrm{G}=(\mathrm{V}, \mathrm{E})$ is $\mathrm{G}^{\mathrm{c}}=\left(\mathrm{V}^{\mathrm{c}}, \mathrm{E}^{\mathrm{c}}\right)$ where $\mathrm{V}^{\mathrm{c}}=\left(\mu_{1}^{\mathrm{c}}, \gamma_{1}^{\mathrm{c}}\right)$ and $E^{c}=\left(\mu_{2}^{c}, \gamma_{2}^{c}\right)$ here $\mu_{1}^{c}=\gamma_{1}^{c}, \mu_{1}^{c}=\gamma_{1}^{c}$ and

$\mu_{2}^{\mathrm{c}}(\mathrm{x}, \mathrm{y})=\mu_{1}(\mathrm{x}) \times \mu_{1}(\mathrm{y})-\mu_{2}(\mathrm{x}, \mathrm{y})$,

$\gamma_{2}^{\mathrm{c}}(\mathrm{x}, \mathrm{y})=\gamma_{1}(\mathrm{x}) \times \gamma_{1}(\mathrm{y})-\gamma_{2}(\mathrm{x}, \mathrm{y})$.

Remark: The complement of a $\mathrm{G}^{\mathrm{c}}$ is $\mathrm{G}$.

Definition2.5 Let $\mathrm{G}_{1}=\left(\mathrm{V}_{1}, \mathrm{E}_{1}\right)$ and $\mathrm{G}_{2}=\left(\mathrm{V}_{2}, \mathrm{E}_{2}\right)$ be a product intuitionistic fuzzy graph. Here $\mathrm{V}_{1}=\left(\mu_{11}, \gamma_{11}\right), \mathrm{E}_{1}=\left(\mu_{12}, \gamma_{12}\right)$, $V_{2}=\left(\mu_{21}, \gamma_{21}\right)$ and $E_{2}=\left(\mu_{22}, \gamma_{22}\right)$. Let $X^{\prime}$ denotes the set of all arcs joining the vertices $\mathrm{V}_{1}$ and $\mathrm{V}_{2}$. We further assume that $\mathrm{V}_{1} \cap \mathrm{V}_{2}=\phi$. Then the join of $\mathrm{G}_{1}$ and $\mathrm{G}_{2}$ is defined as $\left(V_{1}+V_{2}, E_{1}+E_{2}\right)$ where $V_{1}+V_{2}=\left(\mu_{11}+\mu_{21}, \gamma_{11}+\gamma_{21}\right)$ and $\mathrm{V}_{1}+\mathrm{V}_{2}=\left(\mu_{12}+\mu_{22}, \gamma_{12}+\gamma_{22}\right)$ here

$\left(\mu_{11}+\mu_{21}\right)=\mu_{11}(\mathrm{u})$ if $\mathrm{u} \in \mathrm{V}_{1}$

$=\mu_{21}(\mathrm{u})$ if $\mathrm{u} \in \mathrm{V}_{2}$

$\left(\gamma_{11}+\gamma_{21}\right)=\gamma_{11}(u)$ if $u \in V_{1}$

$=\gamma_{21}(\mathrm{u})$ if $\mathrm{u} \in \mathrm{V}_{2}$

$\left(\mu_{12}+\mu_{22}\right)(u, v)=\mu_{12}(u, v)$ if $(u, v) \in E_{1}$

$=\mu_{22}(\mathrm{u}, \mathrm{v})$ if $(\mathrm{u}, \mathrm{v}) \in \mathrm{E}_{2}$

$=\mu_{11}(\mathrm{u}) \times \mu_{21}(\mathrm{v})$ if $(\mathrm{u}, \mathrm{v}) \in \mathrm{X}^{\prime}$

$\left(\gamma_{12}+\gamma_{22}\right)(u, v)=\gamma_{12}(u, v)$ if $(u, v) \in E_{1}$

$=\gamma_{22}(\mathrm{u}, \mathrm{v})$ if $(\mathrm{u}, \mathrm{v}) \in \mathrm{E}_{2}$

$=\gamma_{11}(u) \times \gamma_{21}(v)$ if $(u, v) \in X^{\prime}$.

Proposition 2.2 $G_{1}+G_{2}$ is a product intuitionistic fuzzy sub graph of $\mathrm{G}=(\mathrm{V}, \mathrm{E})$ where and $\mathrm{E}=\mathrm{E}_{1} \cup \mathrm{E}_{2} \cup \mathrm{X}^{\prime}$

Proof: We have to prove that

$\left(\mu_{12}+\mu_{22}\right)(\mathrm{u}, \mathrm{v}) \leq\left(\mu_{11}+\mu_{21}\right)(\mathrm{u}) \times\left(\mu_{11}+\mu_{21}\right)(\mathrm{v}) \rightarrow$

for all $(\mathrm{u}, \mathrm{v}) \in \mathrm{V}$

and $\left(\gamma_{12}+\gamma_{22}\right)(\mathrm{u}, \mathrm{v}) \leq\left(\gamma_{11}+\gamma_{21}\right)(\mathrm{u}) \times\left(\gamma_{11}+\gamma_{21}\right)(\mathrm{v}) \rightarrow$

for all $(\mathrm{u}, \mathrm{v}) \in \mathrm{V}$

Case 1: If $(u, v) \in X_{1}$, then $u, v \in V_{1}$ so that $\left(\mu_{12}+\mu_{22}\right)(\mathrm{u}, \mathrm{v})=\mu_{12}(\mathrm{u}, \mathrm{v}) \rightarrow($ iii)

And $\left(\mu_{11}+\mu_{21}\right)(\mathrm{u}) \times\left(\mu_{11}+\mu_{21}\right)(\mathrm{v})=\mu_{11}(\mathrm{u}) \times \mu_{11}(\mathrm{v}) \rightarrow$

from (iii) and (iv) we get (i)

$\left(\gamma_{12}+\gamma_{22}\right)(\mathrm{u}, \mathrm{v})=\gamma_{12}(\mathrm{u}, \mathrm{v}) \rightarrow$

and $\left(\gamma_{11}+\gamma_{21}\right)(\mathrm{u}) \times\left(\gamma_{11}+\gamma_{21}\right)(\mathrm{v})=\gamma_{11}(\mathrm{u}) \times \gamma_{11}(\mathrm{v}) \rightarrow$

from (v) and (vi) we get (ii)

Therefore we get $G_{1}+G_{2}$ is a product intuitionistic fuzzy sub graph of G.

Similarly we can prove $(\mathrm{u}, \mathrm{v}) \in \mathrm{X}_{1}$.

Case 2: If $(u, v) \in X^{\prime}$ then $u \in V_{1}$ and $v \in V_{2}$. Now $\left(\mu_{12}+\mu_{22}\right)(\mathrm{u}, \mathrm{v}) \leq\left(\mu_{11}(\mathrm{u}) \times \mu_{21}(\mathrm{v}) \quad\right.$ whereas $\left(\mu_{11}+\mu_{21}\right)(\mathrm{u}) \times\left(\mu_{11}+\mu_{21}\right)(\mathrm{v})=\mu_{11}(\mathrm{u}) \times \mu_{21}(\mathrm{v})$ therefore we get $\left(\mu_{12}+\mu_{22}\right)(\mathrm{u}, \mathrm{v})=\left(\mu_{11}+\mu_{21}\right)(\mathrm{u}) \times\left(\mu_{11}+\mu_{21}\right)(\mathrm{v}) \rightarrow \quad$ (vii)

Similarly we get

$\left(\gamma_{12}+\gamma_{22}\right)(\mathrm{u}, \mathrm{v})=\left(\gamma_{11}+\gamma_{21}\right)(\mathrm{u}) \times\left(\gamma_{11}+\gamma_{21}\right)(\mathrm{v}) \rightarrow \quad($ viii $)$

From (vii) and (viii) $\mathrm{G}_{1}+\mathrm{G}_{2}$ is a product intuitionistic fuzzy sub graph of G.Hence proved.

Proposition 2.3 $G_{1}+G_{2}$ is complete if and only if $G_{1}$ and $G_{2}$ are both complete.

Proof: First we are assuming that $\mathrm{G}_{1}$ and $\mathrm{G}_{2}$ are both complete. We will prove that $G_{1}+G_{2}$ is complete.

Case 1: If $(u, v) \in X_{1}$ therefore $u, v \in V_{1}$.we get $\left(\mu_{12}+\mu_{22}\right)(\mathrm{u}, \mathrm{v})=\mu_{12}(\mathrm{u}, \mathrm{v})=\mu_{11}(\mathrm{u}) \times \mu_{11}(\mathrm{v})\left[\right.$ since $\mathrm{G}_{1}$ is complete]

$$
\left(\mu_{11}+\mu_{21}\right)(\mathrm{u}) \times\left(\mu_{11}+\mu_{21}\right)(\mathrm{v})=\mu_{11}(\mathrm{u}) \times \mu_{11}(\mathrm{v})
$$

and $\left(\gamma_{12}+\gamma_{22}\right)(\mathrm{u}, \mathrm{v})=\gamma_{12}(\mathrm{u}, \mathrm{v})=\gamma_{11}(\mathrm{u}) \times \gamma_{11}(\mathrm{v})\left[\right.$ since $\mathrm{G}_{1}$ is complete]

$\left(\gamma_{11}+\gamma_{21}\right)(\mathrm{u}) \times\left(\gamma_{11}+\gamma_{21}\right)(\mathrm{v})=\gamma_{11}(\mathrm{u}) \times \gamma_{11}(\mathrm{v})$

Therefore $\mathrm{G}_{1}+\mathrm{G}_{2}$ is complete.

Similarly we $\operatorname{argue}(\mathrm{u}, \mathrm{v}) \in \mathrm{X}_{2}$ therefore $\mathrm{u}, \mathrm{v} \in \mathrm{V}_{2}$.

Case 2: Suppose $(u, v) \in X^{\prime}$. Then $u \in V_{1}$ and $v \in V_{2}$, we get $\left(\mu_{12}+\mu_{22}\right)(u, v)=\mu_{11}(u) \times \mu_{21}(v)$ whereas $\left(\mu_{11}+\mu_{21}\right)(\mathrm{u}) \times\left(\mu_{11}+\mu_{21}\right)(\mathrm{v})=\mu_{11}(\mathrm{u}) \times \mu_{21}(\mathrm{v})$

Therefore, $\left(\mu_{12}+\mu_{22}\right)(u, v)=\left(\mu_{11}+\mu_{21}\right)(u) \times\left(\mu_{11}(u) \times \mu_{21}\right)(v)$

And $\left(\gamma_{12}+\gamma_{22}\right)(\mathrm{u}, \mathrm{v})=\gamma_{11}(\mathrm{u}) \times \gamma_{21}(\mathrm{v})$ whereas

$\left(\gamma_{11}+\gamma_{21}\right)(u) \times\left(\gamma_{11}+\gamma_{21}\right)(v)=\gamma_{11}(u) \times \gamma_{21}(v)$

Therefore, $\left(\gamma_{12}+\gamma_{22}\right)(\mathrm{u}, \mathrm{v})=\left(\gamma_{11}+\gamma_{21}\right)(\mathrm{u}) \times\left(\gamma_{11}+\gamma_{21}\right)(\mathrm{v})$

Therefore $\mathrm{G}_{1}+\mathrm{G}_{2}$ is complete.

Conversely assume that $G_{1}+G_{2}$ is complete, we will prove $G_{1}$, $\mathrm{G}_{2}$ are complete. First we

$\mathrm{G}_{1}$ complete, we have prove that for al (u, v) $\in \mathrm{E}_{1}$, $\mu_{12}(\mathrm{u}, \mathrm{v})=\mu_{11}(\mathrm{u}) \times \mu_{11}(\mathrm{v})$ and $\gamma_{12}(\mathrm{u}, \mathrm{v})=\gamma_{11}(\mathrm{u}) \times \gamma_{11}(\mathrm{v})$. $\mathrm{G}_{1}+\mathrm{G}_{2}$ is complete therefore

$\left(\mu_{12}+\mu_{22}\right)(\mathrm{u}, \mathrm{v})=\mu_{12}(\mathrm{u}, \mathrm{v}) \rightarrow \quad$ (i) $\quad\left[\operatorname{since}(\mathrm{u}, \mathrm{v}) \in \mathrm{E}_{1}\right]$ and

$\left(\gamma_{12}+\gamma_{22}\right)(\mathrm{u}, \mathrm{v})=\gamma_{12}(\mathrm{u}, \mathrm{v}) \rightarrow$ (ii) $\left[\operatorname{since}(\mathrm{u}, \mathrm{v}) \in \mathrm{E}_{2}\right.$ ] whereas $\left(\mu_{11}+\mu_{21}\right)(\mathrm{u}) \times\left(\mu_{11}+\mu_{21}\right)(\mathrm{v})=\mu_{11}(\mathrm{u}) \times \mu_{11}(\mathrm{v}) \rightarrow$ (iii) and

$\left(\gamma_{11}+\gamma_{21}\right)(\mathrm{u}) \times\left(\gamma_{11}+\gamma_{21}\right)(\mathrm{v})=\gamma_{11}(\mathrm{u}) \times \gamma_{11}(\mathrm{v}) \rightarrow(\mathrm{iv})$

We know that $\left(\mu_{12}+\mu_{22}\right)(\mathrm{u}, \mathrm{v})=\left(\mu_{11}+\mu_{21}\right)(\mathrm{u}) \times\left(\mu_{11}+\mu_{21}\right)(\mathrm{v})$

$\left(\gamma_{12}+\gamma_{22}\right)(\mathrm{u}, \mathrm{v})=\left(\gamma_{11}+\gamma_{21}\right)(\mathrm{u}) \times\left(\gamma_{11}+\gamma_{21}\right)(\mathrm{v})$

using (i), (ii), (iii),(iv) we get $\mu_{12}$ (u, v) $=\mu_{11}$ (u) $\times \mu_{11}$ (v) and $\gamma_{12}(u, v)=\gamma_{11}(u) \times \gamma_{11}(v)$. Therefore $G_{1}$ is complete. Similarly we prove $\mathrm{G}_{2}$ is complete.

Hence proved.

Proposition 2.4 Let $G_{1}$ and $G_{2}$ be product partial intuitionistic fuzzy sub graph, then

$\left(\mu_{11}+\mu_{21}, \mu_{12}+\mu_{22}\right)^{c}=\left(\mu_{11}{ }^{c} \cup \mu_{21}{ }^{c}, \mu_{12}{ }^{c} \cup \mu_{22}{ }^{c}\right)$

$\left(\gamma_{11}+\gamma_{21}, \gamma_{12}+\gamma_{22}\right)^{\mathrm{c}}=\left(\gamma_{11}{ }^{\mathrm{c}} \cup \gamma_{21}{ }^{\mathrm{c}}, \gamma_{12}{ }^{\mathrm{c}} \cup \gamma_{22}{ }^{\mathrm{c}}\right)$

Proof: If $\mathrm{u} \in \mathrm{V}_{1}$ then $\left(\mu_{11}+\mu_{21}\right)^{\mathrm{c}}=\left(\mu_{11}+\mu_{21}\right)(\mathrm{u})=\mu_{11}(\mathrm{u})$ and $\max \left(\mu_{11}{ }^{\mathrm{c}}(\mathrm{u}), \mu_{21}{ }^{\mathrm{c}}(\mathrm{u})=\max \left(\mu_{11}(\mathrm{u}), \mu_{21}(\mathrm{u})\right)=\mu_{11}(\mathrm{u})\right.$

$$
\Rightarrow\left(\mu_{11}+\mu_{21}\right) \quad{ }^{c}(u)=\left(\mu_{11}^{c} \cup \mu_{21}{ }^{c}\right)(u) \text {. }
$$

Similarly $\mathrm{u} \in \mathrm{V}_{2}$.

Suppose $(\mathrm{u}, \mathrm{v}) \in \mathrm{X}_{1}$, then $\mathrm{u}, \mathrm{v} \in \mathrm{V}_{1}$ and

$\left(\mu_{12}+\mu_{22}\right)^{\mathrm{c}}(\mathrm{u}, \mathrm{v})=\left(\mu_{11}+\mu_{21}\right)(\mathrm{u}) \times\left(\mu_{11}+\mu_{21}\right)(\mathrm{v})-\left(\mu_{12}+\mu_{22}\right)$ $(\mathrm{u}, \mathrm{v})$

$$
\begin{aligned}
& =\mu_{11}(\mathrm{u}) \times \mu_{11}(\mathrm{v})-\mu_{12}(\mathrm{u}, \mathrm{v}) \\
& =\mu_{12}{ }^{\mathrm{c}}(\mathrm{u}, \mathrm{v})
\end{aligned}
$$

$\operatorname{Max}\left(\mu_{12}{ }^{\mathrm{c}}(\mathrm{u}, \mathrm{v}), \mu_{21}{ }^{\mathrm{c}}(\mathrm{u}, \mathrm{v})\right)=\mu_{12}{ }^{\mathrm{c}}(\mathrm{u}, \mathrm{v})$

Similarly $(\mathrm{u}, \mathrm{v}) \in \mathrm{X}_{2}$

$$
\Rightarrow\left(\mu_{12}+\mu_{22}\right)^{\mathrm{c}}(\mathrm{u}, \mathrm{v})=\left(\mu_{12}{ }^{\mathrm{c}} \cup \mu_{22}{ }^{\mathrm{c}}\right) \text {. }
$$

Suppose $(\mathrm{u}, \mathrm{v}) \in \mathrm{X}^{\prime}$. Then $\mathrm{u} \in \mathrm{V}_{1}$ and $\mathrm{v} \in \mathrm{V}_{2}$ therefore

$\left(\mu_{12}+\mu_{22}\right)^{\mathrm{c}}(\mathrm{u}, \mathrm{v})=\left(\mu_{11}+\mu_{21}\right)(\mathrm{u}) \times\left(\mu_{11}+\mu_{21}\right)(\mathrm{v})-\left(\mu_{12}+\mu_{22}\right)$ $(\mathrm{u}, \mathrm{v})$

$$
=\mu_{11}(\mathrm{u}) \times \mu_{21}(\mathrm{v})-\left(\mu_{11}(\mathrm{u}) \times \mu_{21}(\mathrm{v})\right)=0
$$

$\operatorname{Max}\left(\mu_{12}{ }^{\mathrm{c}}, \mu_{22}{ }^{\mathrm{c}}\right)=\operatorname{Max}\left(\mu_{12}{ }^{\mathrm{c}}(\mathrm{u}, \mathrm{v}), \mu_{22}{ }^{\mathrm{c}}(\mathrm{u}, \mathrm{v})\right)=0$ [since $\mathrm{u} \in \mathrm{V}_{1}$ and $\mathrm{v} \in \mathrm{V}_{2}$ ]

This implies $\left(\mu_{11}+\mu_{21}, \mu_{12}+\mu_{22}\right)^{c}=\left(\mu_{11}{ }^{c} \cup \mu_{21}{ }^{c}, \mu_{12}{ }^{c} \cup \mu_{22}{ }^{c}\right)$

$\left(\gamma_{11}+\gamma_{21}, \gamma_{12}+\gamma_{22}\right)^{\mathrm{c}}=\left(\gamma_{11}{ }^{\mathrm{c}} \cup \gamma_{21}{ }^{\mathrm{c}}, \gamma_{12}{ }^{\mathrm{c}} \cup \gamma_{22}{ }^{\mathrm{c}}\right)$

Hence proved.

Proposition 2.5 Let $G_{1}$ and $G_{2}$ be product partial intuitionistic fuzzy sub graph, then 


$$
\left(\left(\mu_{11} \cup \mu_{21}\right)^{\mathrm{c}},\left(\mu_{12} \cup \mu_{22}\right)^{\mathrm{c}}\right)=\left(\mu_{11}{ }^{\mathrm{c}}+\mu_{21}{ }^{\mathrm{c}}, \mu_{12}{ }^{\mathrm{c}}+\mu_{22}{ }^{\mathrm{c}}\right)
$$$$
\left(\left(\gamma_{11} \cup \gamma_{21}\right)^{\mathrm{c}},\left(\gamma_{12} \cup \gamma_{22}\right)^{\mathrm{c}}\right)=\left(\gamma_{11}{ }^{\mathrm{c}}+\gamma_{21}{ }^{\mathrm{c}}, \gamma_{12}{ }^{\mathrm{c}}+\gamma_{22}{ }^{\mathrm{c}}\right)
$$

\section{Proof:}

Case 1: If $u \in V_{1}$, then $\left(\mu_{11} \cup \mu_{21}\right)^{c}(u)=\left(\mu_{11} \cup \mu_{21}\right)(u)=\mu_{11}(u)$ $\left(\mu_{11}{ }^{\mathrm{c}}+\mu_{21}{ }^{\mathrm{c}}\right)(\mathrm{u})=\max \left(\mu_{11}(\mathrm{u}), \mu_{21}(\mathrm{u})\right)^{\mathrm{c}}=\max \left(\mu_{11}{ }^{\mathrm{c}}(\mathrm{u}), \mu_{21}{ }^{\mathrm{c}}(\mathrm{v})\right)=$ $\mu_{11}(\mathrm{u})$

This implies $\left(\left(\mu_{11} \cup \mu_{21}\right)^{\mathrm{c}}=\left(\mu_{11}{ }^{\mathrm{c}}+\mu_{21}{ }^{\mathrm{c}}\right)\right.$. similarly we can prove $\mathrm{u} \in \mathrm{V}_{2}$.

Case 2: If $(u, v) \in X_{1}$, then $u, v \in V_{1}$, therefore

$\left(\mu_{12} \cup \mu_{22}\right)^{\mathrm{c}}(\mathrm{u}, \mathrm{v})=\left(\mu_{11} \cup \mu_{21}\right)(\mathrm{u}) \times\left(\mu_{11} \cup \mu_{21}\right)(\mathrm{v})-\left(\mu_{12} \cup\right.$ $\left.\mu_{22}\right)(\mathrm{u}, \mathrm{v})$

$$
\begin{aligned}
& =\mu_{11}(\mathrm{u}) \times \mu_{11}(\mathrm{v})-\mu_{12}(\mathrm{u}, \mathrm{v}) \\
& =\mu_{12} \mathrm{c}(\mathrm{u}, \mathrm{v}) .
\end{aligned}
$$

Case 3: If $(u, v) \in X_{2}$, then $u, v \in V_{2}$, therefore

$\left(\mu_{12} \cup \mu_{22}\right)^{\mathrm{c}}(\mathrm{u}, \mathrm{v})=\left(\mu_{11} \cup \mu_{21}\right)(\mathrm{u}) \times\left(\mu_{11} \cup \mu_{21}\right)(\mathrm{v})-\left(\mu_{12} \cup\right.$ $\left.\mu_{22}\right)(\mathrm{u}, \mathrm{v})$

$$
\begin{aligned}
& =\mu_{21}(\mathrm{u}) \times \mu_{21}(\mathrm{v})-\mu_{22}(\mathrm{u}, \mathrm{v}) \\
& =\mu_{22}{ }^{\mathrm{c}}(\mathrm{u}, \mathrm{v}) .
\end{aligned}
$$

Case 4: If $(u, v) \in X^{\prime}$, then $u \in V_{1}$ and $v \in V_{2}$, therefore $\left(\mu_{12} \cup \mu_{22}\right)^{\mathrm{c}}(\mathrm{u}, \mathrm{v})=\left(\mu_{11} \cup \mu_{21}\right)(\mathrm{u}) \times\left(\mu_{11} \cup \mu_{21}\right)(\mathrm{v})-\left(\mu_{12} \cup\right.$ $\left.\mu_{22}\right)(\mathrm{u}, \mathrm{v})$

$$
\begin{aligned}
& =\mu_{11}(\mathrm{u}) \times \mu_{21}(\mathrm{v}) \\
& \quad\left[\operatorname{since} \mu_{12}(\mathrm{u}, \mathrm{v})=\mu_{22}(\mathrm{u}, \mathrm{v})=0\right] \\
& =\mu_{11}{ }^{\mathrm{c}}(\mathrm{u}) \times \mu_{21} \mathrm{c}(\mathrm{v}) \\
& =\mu_{12}{ }^{\mathrm{c}}+\mu_{22}{ }^{\mathrm{c}}
\end{aligned}
$$

Hence proved.

Proposition 2.6 Let $\mathrm{G}_{1}$ and $\mathrm{G}_{2}$ be product partial intuitionistic fuzzy sub graph, then $\mathrm{G} 1 \times \mathrm{G}_{2}$ be product partial intuitionistic fuzzy sub graph

Proof: $\mathrm{u}_{1}, \mathrm{v}_{1} \in \mathrm{V}_{1}$ and $\mathrm{u}_{2}, \mathrm{v}_{2} \in \mathrm{V}_{2}$, we have

$$
\begin{gathered}
\left(\mu_{12} \times \mu_{22}\right)\left(\left(\mathrm{u} 1, \mathrm{u}_{2}\right),\left(\mathrm{v}_{1}, \mathrm{v}_{2)}\right)=\mu_{12}\left(\mathrm{u}_{1}, \mathrm{v}_{1}\right) \times \mu_{22}\left(\mathrm{u}_{2}, \mathrm{v}_{2}\right)\right. \\
\leq\left[\mu_{11}\left(\mathrm{u}_{1}\right) \times \mu_{11}\left(\mathrm{v}_{1)}\right] \times\left[\mu _ { 2 1 } \left(\mathrm{u}_{2)} \times \mu_{21}\left(\mathrm{v}_{2)}\right]\right.\right.\right. \\
=\left[\mu_{11}\left(\mathrm{u}_{1}\right) \times \mu_{21}\left(\mathrm{u}_{2)}\right] \times\left[\mu _ { 1 1 } \left(\mathrm{v}_{1)} \times \mu_{21}\left(\mathrm{v}_{2)}\right]\right.\right.\right. \\
=\left(\mu_{11} \times \mu_{21}\right)\left(\mathrm{u}_{1}, \mathrm{u}_{2}\right) \times\left(\mu_{11} \times \mu_{21}\right)\left(\mathrm{v}_{1}, \mathrm{v}_{2}\right)
\end{gathered}
$$

Therefore $\left(\mu_{12} \times \mu_{22}\right)\left(\left(\mathrm{u} 1, \mathrm{u}_{2}\right),\left(\mathrm{v}_{1}, \mathrm{v}_{2)}\right)\right) \leq\left(\mu_{11} \times \mu_{21}\right)\left(\mathrm{u}_{1}, \mathrm{u}_{2}\right) \times$ $\left(\mu_{11} \times \mu_{21}\right)\left(\mathrm{v}_{1}, \mathrm{v}_{2}\right)$

similarly we prove

$\left.\left(\gamma_{12} \times \gamma_{22}\right)\left(\left(\mathrm{u}_{1}, \mathrm{u}_{2}\right),\left(\mathrm{v}_{1}, \mathrm{v}_{2}\right)\right) \leq\left(\gamma_{11} \times \gamma_{21}\right)\left(\mathrm{u}_{1}, \mathrm{u}_{2}\right) \times \gamma_{11} \times \gamma_{21}\right)\left(\mathrm{v}_{1}, \mathrm{v}_{2}\right)$ Hence proved.

\section{CONCLUSION}

We are able to find the different types of intuitionistic fuzzy graph and its properties. Further we are try to find the engineering applications of the different types of intuitionist fuzzy graph.

\section{REFERENCES}

[1] A. Rosenfeld. Fuzzy graphs, in: L. A. Zadeh, K. S. Fu, K. Tanaka, M. Shimura (Eds.), Fuzzy Sets and Their Applications to Cognitive and Decision processes, Academic Press, New York, 1975, pp. 77 - 95.

[2] Atanassov KT. Intuitionistic fuzzy sets: theory and applications. Physica, New York,1999.

[3] Harary. F., Graph Theory, Addition Wesley, Third Printing, October 1972

[4] J. N. Mordeson, P. S. Nair, Fuzzy Graphs and Fuzzy Hyper graphs, Physica - Verlag, Heidelberg, 2000.

[5] Parvathi. R and Karunambigai. M.G., Intuitionistic Fuzzy Graphs, Computational Intelligence, Theory and applications, International Conference in Germany, Sept 18, 20, 2006.

[6] Arumugam.S and Velammal.S, Edge Domination in Graphs, Taiwanese Journal of Mathematics, Vol.2, No.2, pp.173-179, June 1998

[7] Ayyaswamy.S, and Natarajan.C, International Journal of Computational and Mathematical sciences, 2010.

[8] Nagoor Gani, A, and Basher Ahamed.M, Order and Size in Fuzzy Graphs, Bulletin of Pure and Applied Science, Vol 22E, 2003; p.145-148

[9] Nagoor Gani, A., and Vadivel.P, Relation between the Parameter of Independent Domination and Irredundance in Fuzzy Graph, International Journal of Algorithms, Computing and Mathematics, Vol 2,2009.

[10] Somasundaram,A.,Somasundaram,S.,1998, Domination in Fuzzy Graphs-I, Pattern Recognition Letters, 19, pp. 787791.

[11] Somasundaram, A., 2004, Domination in Fuzzy Graph-II, Journal of Fuzzy Mathematics 